\title{
Breath holding spells in 91 children and response to treatment with iron
}

\author{
Hilal Mocan, Alisan Yildiran, Fazil Orhan, Erol Erduran
}

\begin{abstract}
To evaluate the prognosis of breath holding spells (BHS) after iron treatment, 91 children ( 56 boys, 35 girls) aged between 6 months and 40 months (median, 17) were followed prospectively for a median of 45 months (range, 6-89). In 49 of the children, the frequency of BHS was less than 10 each month, in 22 it was 10-30 each month, and in 20 more than 30 each month. The spells were cyanotic in 60 children. All patients were evaluated initially and during follow up for haematological indices. Electroencephalographic and electrocardiographic abnormalities were also recorded. Sixty three patients were found to have iron deficiency anaemia and were treated with iron $(6 \mathrm{mg} / \mathrm{kg} /$ day) for three months. Other patients were not given any treatment. After three months, there was a significant difference for correction of cyanotic spells between children who had been treated with iron and those who had not $(84.1 \%$ v $21.4 \%)$. During further follow up, febrile convulsions occurred in 10 children (six were on iron treatment initially). It appears that treating iron deficiency anaemia is effective in reducing the frequency of BHS.

(Arch Dis Child 1999;81:261-262)
\end{abstract}

Keywords: iron deficiency; breath holding spells; anaemia

Breath holding spells (BHS) are a well recognised, common problem affecting about $27 \%$ of healthy children. ${ }^{1}$ The presence of an underlying dysfunctional autonomic nervous system in children with BHS has been proposed by many authors. ${ }^{2}$ Breath holding spells have also been associated with iron deficiency anaemia (IDA). ${ }^{3}$ IDA may lead to adverse effects on oxygen uptake in the lungs and reduce available oxygen to the tissues, including central nervous system tissue. ${ }^{45}$

Several studies have reported abatement of BHS with iron treatment, which may suggest a relation between IDA and BHS. ${ }^{6-8}$

We present the results of our experience in 91 children with BHS who were followed for more than four years.

\section{Patients and methods}

We studied 91 children ( 56 boys, 35 girls) with BHS diagnosed between 1992 and 1995. Their ages at diagnosis ranged from 6 to 30 months. Diagnosis of BHS was made clinically by a paediatrician based on the history given by the mothers and observation of the spells. Spells were defined as the child's breathing stopping in expiration after a deep inspiration during crying. ${ }^{9}$ The spells were classified into cyanotic, pallid, and mixed (when there was no clear distinction between cyanosis and pallor according to the skin colour change during episodes). Patients with a haemoglobin level of $\leqslant 70 \mathrm{~g} / \mathrm{l}$, a history of febrile convulsions or epilepsy, current treatment with anticonvulsant medications, a clinically identified mental disability, or severe malnutrition were excluded from our study.

A detailed medical and family history was taken before entry. We recorded type and frequency of the attacks, and whether parents were consanguineous. Haemoglobin concentration, mean corpuscular volume (MCV), serum iron (SI), total iron binding capacity (TIBC), and transferrin saturation (TS) were determined for each patient initially and repeated three monthly during follow up. Blood sugar and calcium concentrations were recorded and electroencepholography (EEG), electrocardiography (ECG), and a skull $x$ ray were carried out.

The 63 patients with BHS and concomitant IDA (haemoglobin levels < $105 \mathrm{~g} / 1$ and MCV values $<75 \mathrm{fl})^{10}$ were treated with ferrous sulphate solution $6 \mathrm{mg} / \mathrm{kg} /$ day orally for three months. In the remaining 28 patients without IDA, treatment was not given. The clinical condition of the patient and the number of attacks were recorded at each visit.

The frequency of attacks was recorded according to the information given by the mother. At the end of the three months the response to the treatment was evaluated. We defined the response as follows: "complete remission", the attacks disappeared completely; "partial remission", $a \geqslant 50 \%$ reduction in the attacks; and "no response", no change in the number of attacks.

Table 1 Initial clinical characteristics of the patients with and without iron deficiency anaemia (IDA)

\begin{tabular}{lll}
\hline & $\begin{array}{l}I D A \\
(n=63)\end{array}$ & $\begin{array}{l}\text { Non-IDA } \\
(n=28)\end{array}$ \\
\hline Sex & & \\
$\quad$ Male & 41 & 15 \\
$\quad$ Female & 22 & 13 \\
Median age at onset (months) & 17 & 14 \\
Frequency of spells/month & & \\
$\quad<10$ & 32 & 17 \\
10-30 & 16 & 6 \\
$>30$ & 15 & 5 \\
Type of attack & & \\
$\quad$ Cyanotic & 42 & 18 \\
$\quad$ Pallid & 6 & 3 \\
$\quad$ Mixed & 15 & 7 \\
Consanguinity & 5 & 3 \\
Positive family history & 8 & 1 \\
\hline
\end{tabular}


Table 2. Mean values of blood indexes in the patients with and withour iron deficiency anaemia (IDA)

\begin{tabular}{llll}
\hline & IDA $(n=63)$ & Non-IDA $(n=28)$ & $p$ Value \\
\hline Haemoglobin (g/l) & $99(10.5)$ & $11.1(1.69)$ & $<0.01$ \\
MCV (f) & $68(7.32)$ & $73.2(8.28)$ & $<0.01$ \\
SI (mg/dl) & $34.7(17.41)$ & $66.4(34.16)$ & $<0.01$ \\
TIBC (mg/dl) & $414.4(67.9)$ & $373.4(41.6)$ & $<0.05$ \\
TS $(\%)$ & $9.1(3.71)$ & $20.1(6.03)$ & $<0.05$ \\
Remission (n (\%)) & & & \\
$\quad$ Complete & $32(50.7)$ & $2(7.1)$ & $<0.02$ \\
$\quad$ Partial & $21(33.3)$ & $4(14.3)$ & $<0.02$ \\
$\quad$ None or minimal & $10(16)$ & $22(78.6)$ & \\
\hline
\end{tabular}

Values are mean (SD) except where stated.

$\mathrm{MCV}$, mean corpuscular volume; SI, serum iron; TIBC, total iron binding capacity; TS, transferrin saturation.
Our data suggest that iron may be crucial in the treatment of BHS because IDA may be the main underlying defect.

It is not known how iron deficiency leads to BHS. It may involve the role of iron in catecholamine metabolism and the functioning of enzymes and neurotransmitters in the central nervous system. ${ }^{10}$ The correction of spells during treatment with iron may be related to the functional restoration of these neurotransmitters. However, iron deficiency is not the only factor responsible for BHS because not all children with BHS were iron deficient at baseline. In addition, we cannot explain why some children with BHS who were not iron deficient responded to iron treatment.

We have hypothesised that the clinical and haematological picture of BHS relates to the interactions of cerebral erythropoietin, nitric oxide and interleukin $1 .{ }^{11}{ }^{12} \mathrm{We}$ suggest that increased brain erythropoietin production has a protective effect during BHS, but where this does not compensate for the severity of anoxic spells, then seizures may develop. Possible neuronal cell death and a protective effect of erythropoietin should be determined in the future for potentially hypoxic conditions such as BHS.

As reported previously, a low serum ferritin may be the earliest laboratory finding of IDA. ${ }^{813}$ We were unable to evaluate ferritin concentrations in all of our patients.

We conclude that children with BHS require investigation and treatment, where appropriate, for iron deficiency anaemia and that anticonvulsants are not the treatment of choice.

Blood indexes of all patients with IDA became normal after iron treatment.

ECGs were all normal, but in 18 patients slight or moderate EEG abnormalities were recorded. Two patients with slight paroxysmal activity on EEG developed febrile convulsions on follow up. Ten of the 91 patients with BHS developed febrile convulsions (six had been treated with iron initially); all had typical BHS episodes initially.

\section{Discussion}

An association between anaemia and BHS has been recognised previously, but published reports are few. Holowach et al demonstrated that children with severe BHS had significantly lower haemoglobin values than children in a control group. ${ }^{3}$ Bhatia et al also found that children with BHS had significantly lower haemoglobin and serum iron values, a lower percentage of transferrin saturation, and higher TIBC than those of controls. ${ }^{6}$ Colina and Abelson reported the resolution of BHS in two children with correction of anaemia. ${ }^{7}$ Daoud et al demonstrated recently that iron treatment is effective in the treatment of BHS and that iron deficient children are more likely to benefit from such treatment.
1 Bridge EM, Livingston S, Tietze C. Breath-holding spells: their relationship to syncope, convulsions, and other phenomena. F Pediatr 1943;23:539-61.

2 Dimario FJ, Chee CM, Berman PH. Pallid breath-holding spells: evaluation of the autonomic nervous system. Clin spells: evaluation of the
Pediatr 1990;29:17-24.

3 Holowach J, Thurston DL. Breath-holding spells and anaemia. N Engl f Med 1963;268:21-3.

4 Samuels MP, Talber DG, Southall DP. Cyanotic breathholding and sudden death. Arch Dis Child 1991;66:257-8. 5 Poets CF, Samuels MP, Wardrop CAJ, Picton-Jones E, Southall DP. Reduced hemoglobin levels in infants presenting with apparent life treating events: a retrospective investigation. Acta Pediatr 1992;81:319-21.

6 Bhatia MS, Singhal PK, Dhar NK, Nigam VR, Malik SC, Mullick DN. Breath-holding spells: an analysis of 50 cases. Indian f Pediatr 1990;27:1073-9.

7 Colina KF, Abelson HT. Resolution of breath-holding spells with treatment of concomittant anaemia. $\mathcal{F}$ Pediatr 1995; 126:395-7.

8 Daoud AS, Batieha A, Al-Sheyyab M, Ebuekteish F, Hijazi S. Effectiveness of iron therapy on breath-holding spells. $\mathcal{F}$ Pediatr 1997;130:547-50.

9 De Lorenzo RJ. The epilepsies. In: Bradley WG, Daroff, RB, eds. Neurology in clinical practice, Vol. II. Boston: Butterworth and Heinemann, 1991:1469.

10 Oski FA, Honig AS. The effects on the developmental scores of iron-deficient infants. F Pediatr 1978;92:21-5.

11 Mocan MC, Mocan H, Aslan Y, Erduran E. Iron therapy in Pediatr 1998;133:583-4.

12 Masuda S, Okano M, Yamagishi K, Nagao M, Ueda M, Sasaki R. A novel site of erythropoetin production: oxygendependent production in cultured rat astrocytes. $\mathscr{f}$ Biol Chem 1994;269:19488-93.

13 Finch CA, Vellotti V, Stray S, et al. Plasma ferritin determination as a diagnostic tool. West $\mathcal{F}$ Med 1986;145:657-63. breath-holding spells and cerebral erythropoetin [letter]. $\mathcal{F}$ 